\title{
Use of imaging tests after primary treatment of thyroid cancer in the United States: population based retrospective cohort study evaluating death and recurrence
}

\author{
Mousumi Banerjee, ${ }^{1,2}$ Jaime L Wiebel, ${ }^{3}$ Cui Guo, ${ }^{1}$ Brittany Gay, ${ }^{4}$ Megan R Haymart2,4,5
}

1Department of Biostatistics, University of Michigan, Ann Arbor, MI, USA

${ }^{2}$ Institute for Healthcare Policy and Innovation (IHPI), University of Michigan, Ann Arbor, MI 48109, USA

${ }^{3}$ Endocrine Associates of Dallas, Dallas, TX, USA

${ }^{4}$ Division of Metabolism, Endocrinology, and Diabetes, University of Michigan, Ann Arbor, MI, USA

${ }^{5}$ Division of Hematology/ Oncology, University of Michigan, Ann Arbor, MI, USA

Correspondence to: M R Haymart meganhay@med.umich.edu

Cite this as: BMJ 2016;354:i3839 http://dx.doi.org/10.1136/bmj.i3839

Accepted: 26 June 2016

\begin{abstract}
OBJECTIVE

To determine whether the use of imaging tests after primary treatment of differentiated thyroid cancer is associated with more treatment for recurrence and fewer deaths from the disease.
\end{abstract}

DESIGN

Population based retrospective cohort study.

SETTING

Surveillance Epidemiology and End Results-Medicare database in the United States.

\section{PARTICIPANTS}

28220 patients diagnosed with differentiated thyroid cancer between 1998 and 2011. The study cohort was followed up to 2013 , with a median follow-up of 69 months.

\section{MAIN OUTCOME MEASURES}

Treatment for recurrence of differentiated thyroid cancer (additional neck surgery, additional radioactive iodine treatment, or radiotherapy), and deaths due to differentiated thyroid cancer. We conducted propensity score analyses to assess the relation between imaging (neck ultrasound, radioiodine scanning, or positron emission tomography (PET) scanning) and treatment for recurrence (logistic model) and death (Cox proportional hazards model).

RESULTS

From 1998 until 2011, we saw an increase in incident cancer (rate ratio 1.05, 95\% confidence interval

\section{WHAT IS ALREADY KNOWN ON THIS TOPIC}

Over the past two decades, the incidence of thyroid cancer has risen; most of this rise is secondary to an increase in low risk disease (that is, disease that will not result in death)

During this same period, the use of imaging after primary thyroid cancer treatment has also increased

The relation between imaging after primary treatment and thyroid cancer outcomes has not been assessed

\section{WHAT THIS STUDY ADDS}

In a cohort of 28220 patients with differentiated thyroid cancer diagnosed between 1998 and 2011 and included in the SEER-Medicare database, there was a substantial rise in incident cancer, imaging after primary treatment, and treatment for recurrence. There was no significant change in death rate

Use of neck ultrasounds, radioiodine scans, and positron emission tomography scans were associated with treatment for recurrence, but only the use of radioiodine scans was associated with improved disease specific survival In light of the growing incidence of low risk thyroid cancer and the paradoxical rise in imaging after primary treatment, this study provides the foundation needed to reassess thyroid cancer surveillance patterns and to curb unnecessary imaging

1.05 to 1.06$)$, imaging $(1.13,1.12$ to 1.13$)$, and treatment for recurrence $(1.01,1.01$ to 1.02$)$; the change in death rate was not significant. In multivariable analysis, use of neck ultrasounds increased the likelihood of additional surgery (odds ratio 2.30, 95\% confidence interval 2.05 to 2.58 ) and additional radioactive iodine treatment (1.45, 1.26 to 1.69). Radioiodine scans were associated with additional surgery (odds ratio 3.39, $95 \%$ confidence interval 3.06 to 3.76 ), additional radioactive iodine treatment $(17.83,14.49$ to 22.16$)$, and radiotherapy $(1.89,1.71$ to 2.10$)$. Use of PET scans was associated with additional surgery (odds ratio $2.31,95 \%$ confidence interval 2.09 to 2.55 ), additional radioactive iodine treatment $(2.13,1.89$ to 2.40$)$, and radiotherapy $(4.98,4.52$ to 5.49$)$. Use of neck ultrasounds or PET scans did not significantly affect disease specific survival (hazard ratio 1.14, 95\% confidence interval 0.98 to 1.27 , and $0.91,0.77$ to 1.07 , respectively). However, radioiodine scans were associated with an improved disease specific survival (hazard ratio 0.70, 95\% confidence interval 0.60 to 0.82 ).

\section{CONCLUSIONS}

The marked rise in use of imaging tests after primary treatment of differentiated thyroid cancer has been associated with an increased treatment for recurrence. However, with the exception of radioiodine scans in presumed iodine avid disease, this association has shown no clear improvement in disease specific survival. These findings emphasize the importance of curbing unnecessary imaging and tailoring imaging after primary treatment to patient risk.

\section{Introduction}

Differentiated thyroid cancer has been increasing in incidence for several decades and is expected to be the fourth leading cancer diagnosis by the year $2030 .{ }^{12}$ Most of this rise in incidence is explained by diagnoses of small, low risk cancers. ${ }^{34}$ These low risk cancers are associated with an excellent long term survival; disease specific survival at 10 years for patients diagnosed with low risk thyroid cancer is between $96 \%$ to $100 \% .{ }^{5}$ Yet, despite this increase in low risk cancers, our previous work found that the use of imaging tests after primary treatment of thyroid cancer is rising. ${ }^{6}$ The reason for this rise in imaging is unknown, but because risk of death from thyroid cancer is low, increased use of imaging could be due to growing concern for the risk of recurrence, which ranges from $1 \%$ to $68 \% .^{78}$ When thyroid cancer recurrence occurs, treatment options include repeat neck surgery, radioactive iodine for iodine avid recurrence, and radiation for unresectable disease. However, these treatments are not without 
risks, ${ }^{9-16}$ and the relation between imaging, treatment for recurrence, and survival remains unknown.

Owing to the lack of evidence linking imaging after primary treatment of thyroid cancer to improvement in outcome, the standard surveillance strategy for patients with differentiated thyroid cancer continues to evolve. ${ }^{17-19}$ However, despite no evidence for survival benefit, there are costs, potential radiation exposure, and potential downstream treatment risks associated with imaging after primary treatment.9-11 1516 Therefore, the rising use of imaging after primary treatment necessitates further inquiry into the effects on patient outcomes, including treatment for recurrence and disease specific survival.

We hypothesize that undergoing imaging after primary treatment of thyroid cancer will be associated with more treatment for recurrence and improved survival. We examine trends over time for diagnosis of differentiated thyroid cancer, use of imaging after primary treatment, treatment for recurrence, and death in patients with the disease. We then determine whether undergoing an imaging test is associated with an increased likelihood of treatment for recurrence or improved disease specific survival.

\section{Methods}

Data source and study population

The Surveillance, Epidemiology, and End Results (SEER) Program of the National Cancer Institute provides population based data on cancer in the USA. SEER cancer registries include about $30 \%$ of the US population. ${ }^{20}$ From 1991, data from SEER were linked with Medicare reimbursement claims to create a dataset that included information on services provided before and after cancer diagnosis. For this study, all patients from the SEER-Medicare linked database who were diagnosed with differentiated thyroid cancer (defined as papillary, follicular, or Hürthle cell tumor histology) between 1 January 1998 and 31 December 2011 were identified and included in the study cohort..$^{21}$ In total, 28220 patients were included. The study used linked longitudinal data that follows treatment episode over time. The study cohort was followed up to 2013, with a median follow-up of 69 months.

\section{Measures}

SEER collects data on patient demographics, tumor site, stage, and treatment. For patients with well differentiated thyroid cancer, the analysis included information on age, SEER defined race or ethnicity (included to correct for racial disparities), median household income by geographical region, percentage of population over age 25 years of the geographical region with only a high school diploma, tumor size $(<1.0 \mathrm{~cm}, 1.0-1.9 \mathrm{~cm}$, 2.0-3.9 cm, and $>4 \mathrm{~cm}$ ), tumor histology, and SEER stage at diagnosis. The type of initial surgery (characterized as no surgery, lobectomy, or total thyroidectomy) and presence or absence of initial lymph node resection were included.

SEER-Medicare data were used to obtain information on imaging after primary treatment. We used Current
Procedure Terminology (CPT) codes to identify the type of imaging used. Neck ultrasounds were identified by CPT code 76536 . Radioiodine scans were identified by CPT codes 78015, 78016, 78017, 78018, 78020, 78800, 78801, 78802, and 78804. We excluded the first billing of radioiodine scans done, because we thought that they were likely to be part of initial staging and not used for surveillance. Positron emission tomography (PET) scans were identified by CPT codes 78810, 78811, 78812, 78813, 78814, 78815, and 78816.

We also used SEER-Medicare CPT codes to identify treatments for recurrence. Codes used to identify repeat neck surgeries were 38510, 38520, 38720, 38724, and 60260 . Codes used to identify radioactive iodine treatment were 79005,79030 , and 75035 . We omitted the first billing for radioactive iodine treatment that was performed to avoid including ablation that was done as part of primary treatment for differentiated thyroid cancer. The CPT codes used to identify radiotherapy were 77261,77262 , and 77263.

The mortality data reported by SEER were provided by the National Cancer for Health Statistics. ${ }^{2022}$ SEER uses algorithms to process causes of death from the death certificate in order to identify a single, disease specific, underlying cause of death. ${ }^{20}$ SEER disease specific survival is defined as the time interval from diagnosis to death from differentiated thyroid cancer or time of censoring.

\section{Statistical analysis}

We analyzed baseline patient characteristics, including the proportion of patients who underwent neck ultrasounds, radioiodine scans, and PET scans. To investigate trends over time, the number of diagnoses, imaging tests (a composite of neck ultrasounds, radioiodine scans, and PET scans), treatments for recurrence (a composite of repeat neck surgery, additional radioactive iodine treatment, and radiotherapy), and disease specific deaths were obtained for each year from 1998 to 2011. We used Poisson regression to model the incidence of differentiated thyroid cancer, use of imaging tests, and treatment for recurrence over time. The denominator for incidence was the US standard population ${ }^{20}$ and the denominators for imaging, treatment of recurrence, and death were the number of patients at risk for the specific year.

To address bias due to potential differences between the imaged and non-imaged groups in terms of patient and disease characteristics, we used propensity score based analysis as a technique for quasi-randomization. Use of a specific imaging modality was analyzed as a dichotomous variable (eg, whether the patient had a neck ultrasound or not, radioiodine scan or not, and PET scan or not). In the first step of the analyses, we estimated propensity scores based on the multivariable logistic regression model of the imaging indicator variable. Observed covariates were age, sex, race, education, income, SEER stage at diagnosis, histology, tumor size, initial surgery, and lymph nodes resection. The propensity score for an individual-defined as the conditional probability of being imaged given the 
individual's covariates-has been shown to serve as a balancing score, and can be used to reduce bias due to any covariate imbalance. ${ }^{23}$ Once estimated, the propensity scores were therefore used in a second step model through regression adjustment. The second step model

\begin{abstract}
Table 1 | Demographics of patient population with thyroid cancer $(n=28220)$, based on
\end{abstract} SEER-Medicare data in 1998-2011

\begin{tabular}{|c|c|c|c|}
\hline \multirow[b]{2}{*}{ Characteristics } & \multicolumn{3}{|l|}{ № (\%) } \\
\hline & $\begin{array}{l}\text { Overall } \\
(n=28220)\end{array}$ & $\begin{array}{l}\text { Additional treatment } \\
(\mathrm{n}=6502)\end{array}$ & $\begin{array}{l}\text { Disease specific } \\
\text { death }(n=1155)\end{array}$ \\
\hline Age (years; median (range)) & $65(21-101)$ & $69(21-97)$ & $73(28-98)$ \\
\hline \multicolumn{4}{|l|}{ Sex } \\
\hline Male & $8383(29.7)$ & $2308(35.5)$ & $455(39.4)$ \\
\hline Female & $19837(70.3)$ & $4194(64.5)$ & $700(60.6)$ \\
\hline \multicolumn{4}{|l|}{ Race } \\
\hline White & $22387(79.3)$ & $5235(80.5)$ & $889(77.0)$ \\
\hline Black & $2231(7.9)$ & $415(6.4)$ & $67(5.8)$ \\
\hline Other & $3411(12.1)$ & $836(12.9)$ & $199(17.2)$ \\
\hline Missing data & $191(0.7)$ & $16(0.2)$ & $0(0.0)$ \\
\hline \multicolumn{4}{|c|}{ Education level up to high school only* } \\
\hline$<20 \%$ & $12146(43.0)$ & $2707(41.6)$ & $488(42.3)$ \\
\hline $20-29.9 \%$ & $6142(21.8)$ & $1438(22.1)$ & $265(22.9)$ \\
\hline$\geq 30 \%$ & $6982(24.7)$ & $1780(27.4)$ & $315(27.3)$ \\
\hline Missing data & $2950(10.5)$ & $577(8.9)$ & $87(7.5)$ \\
\hline \multicolumn{4}{|l|}{ Median household incomet } \\
\hline$<\$ 35000$ & $13139(46.6)$ & $3257(50.1)$ & $589(51.0)$ \\
\hline$\$ 35000-59999$ & $10227(36.2)$ & $2251(34.6)$ & $423(36.6)$ \\
\hline$\geq \$ 60000$ & $1904(6.7)$ & $417(6.4)$ & $56(4.9)$ \\
\hline Missing data & $2950(10.5)$ & $577(8.9)$ & $87(7.5)$ \\
\hline \multicolumn{4}{|l|}{ Stage of disease } \\
\hline Localized & $18084(64.1)$ & $3055(47.0)$ & $170(14.7)$ \\
\hline Regional & $8720(30.9)$ & $2746(42.2)$ & $531(46.0)$ \\
\hline Distant & $1416(5.0)$ & $701(10.8)$ & $454(39.3)$ \\
\hline \multicolumn{4}{|l|}{ Histology } \\
\hline Papillary & $24537(86.9)$ & $5287(81.3)$ & $836(72.4)$ \\
\hline Hürthle cell & $1377(4.9)$ & $463(7.1)$ & $104(9.0)$ \\
\hline Follicular & $2306(8.2)$ & $752(11.6)$ & $215(18.6)$ \\
\hline \multicolumn{4}{|l|}{ Tumor size (in diameter) } \\
\hline$<1 \mathrm{~cm}$ & $9051(32.1)$ & $1350(20.8)$ & $77(6.7)$ \\
\hline $1-1.9 \mathrm{~cm}$ & $6488(23.0)$ & $1354(20.8)$ & $98(8.5)$ \\
\hline $2-3.9 \mathrm{~cm}$ & $6411(22.7)$ & $1717(26.4)$ & $271(23.4)$ \\
\hline$>4 \mathrm{~cm}$ & $3808(13.5)$ & $1374(21.1)$ & $432(37.4)$ \\
\hline Missing data & $2462(8.7)$ & 707 (10.9) & $277(24.0)$ \\
\hline \multicolumn{4}{|l|}{ Surgery } \\
\hline No surgery & 1154 (4.1) & $316(4.9)$ & $214(18.5)$ \\
\hline Lobectomy & $5317(18.8)$ & $1057(16.2)$ & $127(11.0)$ \\
\hline Total thyroidectomy & $21659(76.8)$ & $5098(78.4)$ & 807 (69.9) \\
\hline Missing data & $90(0.3)$ & $31(0.5)$ & $7(0.6)$ \\
\hline \multicolumn{4}{|l|}{ Lymph nodes resected } \\
\hline Yes & $10180(36.1)$ & $2824(43.4)$ & $544(47.1)$ \\
\hline No & $17933(63.5)$ & $3656(56.2)$ & $597(51.7)$ \\
\hline Missing data & $107(0.4)$ & $22(0.4)$ & $14(1.2)$ \\
\hline \multicolumn{4}{|l|}{ Neck ultrasound } \\
\hline Yes & $16005(56.7)$ & $4916(75.6)$ & $561(48.6)$ \\
\hline No & $12215(43.3)$ & $1586(24.4)$ & $594(51.4)$ \\
\hline \multicolumn{4}{|l|}{ Radioiodine scan } \\
\hline Yes & $6753(23.9)$ & $3247(49.9)$ & $358(31.0)$ \\
\hline No & $21467(76.1)$ & $3255(50.1)$ & $797(69.0)$ \\
\hline \multicolumn{4}{|l|}{ PET scan } \\
\hline Yes & $4207(14.9)$ & $2359(36.3)$ & $323(28.0)$ \\
\hline No & $24013(85.1)$ & $4143(63.7)$ & $832(72.0)$ \\
\hline
\end{tabular}

*Proportion of patients aged $\geq 25$ years in geographical region with only high school education.

tMedian household income by geographical region. $\$ 1.00=£ 0.77 ; € 0.91$. for investigating the association between use of imaging and undergoing treatment for recurrence was based on multivariable logistic regression of each treatment variable: repeat neck surgery (yes $v$ no), additional treatment with radioactive iodine (yes $v$ no) and radiotherapy (yes $v$ no).

We used Cox proportional hazards regression to investigate the association between use of imaging and disease specific survival. Once again, propensity scores from the first step model described above were used in the second step Cox model through regression adjustment.

Of 28220 patients in the study cohort, $191(0.7 \%)$ had missing information on race, 2950 (10.5\%) had missing information on high school education, 2950 (10.5\%) had missing data on median household income, 2462 (8.7\%) had missing tumor size, $90(0.3 \%)$ had missing surgery, and $107(0.4 \%)$ had missing information on lymph node resection. There were no significant differences in sociodemographics, tumor characteristics, and treatment between the patients with complete data and those with missing data in at least one covariate. Hence, for logistic regression and Cox proportional hazards regression, primary analysis was based on patients with a complete set of information ( $n=22770)$. Additionally, as a sensitivity analysis, we repeated the logistic regression and Cox proportional hazards regression by coding the missing data for each covariate in a separate category by itself. This way, we were able to rerun the analyses on the entire cohort $(n=28220)$. The results were similar. All statistical analyses used SAS 9.4 and R 3.2.4.

\section{Patient involvement}

No patients were involved in setting the research question or the outcome measures, nor were they involved in developing plans for design or implementation of the study. No patients were asked to advise on interpretation or writing up of results. There are no plans to disseminate the results of the research to study participants or the relevant patient community.

\section{Results}

Table 1 describes the demographics of the study population. Overall, $64.1 \%$ of the study population had localized disease, with $32.1 \%$ having tumors less than $1.0 \mathrm{~cm}$ in diameter. Consistent with other studies of differentiated thyroid cancer, $86.9 \%$ of the diagnoses were papillary thyroid cancer and $70.3 \%$ of patients were women. Overall, there was a substantial utilization of imaging after primary treatment, with $56.7 \%$ of patients undergoing at least one neck ultrasound, $23.9 \%$ having a radioiodine scan, and $14.9 \%$ undergoing a PET scan.

Based on the Poisson regression model to account for the increasing size of the population at risk over time (from 1998 to 2011), there was a significant increase in incident cancer (rate ratio 1.05, 95\% confidence interval 1.05 to 1.06$)$, imaging $(1.13,1.12$ to 1.13$)$, and treatment of recurrence (1.01, 1.01 to 1.02; fig 1). Use of imaging rose the most substantially. Change in death rate was not statistically significant (0.98, 0.96 to 1.00$)$. Trends in 
rise of specific treatments after recurrence were 1.02 (1.01 to 1.03) for additional radioactive iodine treatment, 1.01 (0.99 to 1.02) for additional neck surgery, and 1.01 (0.99 to 1.02) for radiotherapy (fig 2).

Table 2 shows the relation between imaging test (neck ultrasound, radioiodine scan, and PET scan) and treatment for recurrence (additional surgery, additional

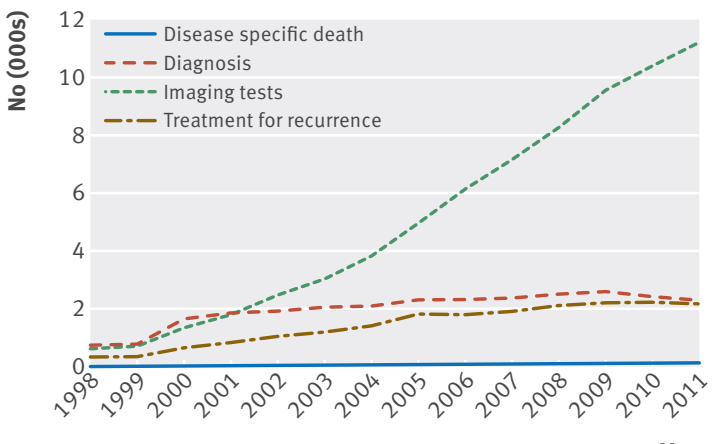

Fig 1 | Trends in thyroid cancer diagnosis, use of imaging, treatment for recurrence, and death from thyroid cancer, based on SEER-Medicare data in 1998-2011. Imaging includes neck ultrasounds, radioiodine scans, and PET scans. Treatments for recurrence include additional neck surgery, additional radioactive iodine treatment, and radiotherapy. Disease specific death is low but not zero

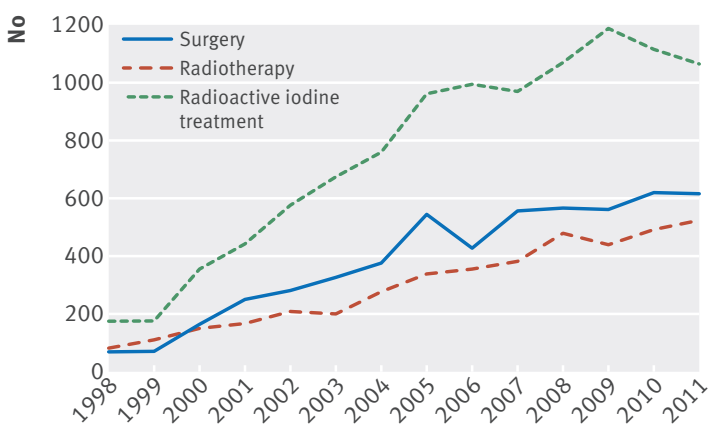

Year

Fig 2 | Trends in use of specific treatments for recurrence (additional neck surgery, additional radioactive iodine treatment, and radiotherapy) in patients with thyroid cancer, based on SEER- Medicare data in 1998-2011 radioactive iodine treatment, and radiotherapy) after controlling for patient and tumor characteristics (patient age, sex, race, education, income, stage of disease, histology, tumor size, type of surgery, and lymph nodes resected). Undergoing ultrasound increased the likelihood of additional surgery (odds ratio 2.30, 95\% confidence interval 2.05 to 2.58 ) and additional radioactive iodine treatment $(1.45,1.26$ to 1.69$)$. We saw no significant relation between use of neck ultrasound and subsequent treatment with radiotherapy (1.08, 0.97 to 1.20). Radioiodine scans were associated with additional surgery (3.39, 3.06 to 3.76), radioactive iodine treatment (17.83, 14.49 to 22.16), and radiotherapy (1.89, 1.71 to 2.10). PET scans were associated with additional surgery (2.31, 2.09 to 2.55), radioactive iodine treatment $(2.13,1.89$ to 2.40$)$, and radiotherapy ( $4.98,4.52$ to 5.49$)$.

A total of 1155 (4.1\%) patients from the study cohort died from thyroid cancer. Results of the multivariable Cox proportional hazards model are shown in table 3. Undergoing neck ultrasound or PET scans did not significantly affect disease specific survival (hazard ratio $1.14,95 \%$ confidence interval 0.98 to 1.27 and $0.91,0.77$ to 1.07 , respectively); however, radioiodine scans were associated with an improved disease specific survival (0.70, 0.60 to 0.82$)$.

\section{Discussion \\ Principal findings}

Relative to the incident cases of differentiated thyroid cancer, the use of imaging after primary treatment has grown dramatically from 1998 to 2011. This substantial increase in imaging after primary treatment is associated with more diagnoses of recurrence, but no significant improvement in disease specific survival, except with use of radioiodine scans. This finding suggests that imaging after primary treatment could lead to detection of more recurrence and subsequent treatment of recurrence, but in many cases without a beneficial effect on disease specific survival.

In our study, use of neck ultrasound was associated with a significant increase in the likelihood of recurrence treatment with surgery and with radioactive iodine treatment. However, neck ultrasound was not associated with improved disease specific survival. It is well known that most thyroid cancer recurrence is

\begin{tabular}{|c|c|c|c|c|c|c|}
\hline \multirow[b]{2}{*}{ Characteristic } & \multicolumn{2}{|l|}{ Repeat neck surgery } & \multicolumn{2}{|l|}{ Radioactive iodine treatment } & \multicolumn{2}{|l|}{ Radiotherapy } \\
\hline & Adjusted odds ratio $(95 \% \mathrm{Cl})$ & $P$ & Adjusted odds ratio $(95 \% \mathrm{Cl})$ & $P$ & Adjusted odds ratio $(95 \% \mathrm{Cl})$ & $P$ \\
\hline \multicolumn{7}{|l|}{ Neck ultrasound } \\
\hline No & 1 (reference) & \multirow{2}{*}{$<0.001$} & 1 (reference) & \multirow{2}{*}{$<0.001$} & 1 (reference) & \multirow{2}{*}{0.17} \\
\hline Yes & $2.30(2.05$ to 2.58$)$ & & $1.45(1.26$ to 1.69$)$ & & 1.08 (0.97 to 1.20$)$ & \\
\hline \multicolumn{7}{|c|}{ Radioiodine (iodine 131) scan } \\
\hline No & 1 (reference) & \multirow{2}{*}{$<0.001$} & 1 (reference) & \multirow{2}{*}{$<0.001$} & 1 (reference) & \multirow{2}{*}{$<0.001$} \\
\hline Yes & 3.39 (3.06 to 3.76 ) & & 17.83 (14.49 to 22.16$)$ & & 1.89 (1.71 to 2.10$)$ & \\
\hline \multicolumn{7}{|l|}{ PET scan } \\
\hline No & 1 (reference) & \multirow{2}{*}{$<0.001$} & 1 (reference) & \multirow{2}{*}{$<0.001$} & 1 (reference) & \multirow{2}{*}{$<0.001$} \\
\hline Yes & 2.31 (2.09 to 2.55$)$ & & 2.13 (1.89 to 2.40$)$ & & $4.98(4.52$ to 5.49$)$ & \\
\hline
\end{tabular}

Analysis based on patients with complete set of information ( $n=22770)$. Adjusted odds ratios and $95 \%$ confidence intervals based on propensity score analyses. Model adjusted for age, sex, race, education, income, stage of disease, histology, tumor size, surgery type, and lymph nodes resected. 


\begin{tabular}{|c|c|c|}
\hline \multirow[b]{2}{*}{ Characteristic } & \multicolumn{2}{|c|}{ Disease specific survival } \\
\hline & $\begin{array}{l}\text { Adjusted hazard } \\
\text { ratio }(95 \% \mathrm{CI})\end{array}$ & $\mathbf{P}$ \\
\hline \multicolumn{3}{|c|}{ Neck ultrasound } \\
\hline No & 1 (reference) & \multirow{2}{*}{0.087} \\
\hline Yes & 1.14 (0.98 to 1.27$)$ & \\
\hline \multicolumn{3}{|c|}{ Radioiodine (iodine 131) scan } \\
\hline No & 1 (reference) & \multirow{2}{*}{$<0.001$} \\
\hline Yes & $0.70(0.60$ to 0.82$)$ & \\
\hline \multicolumn{3}{|l|}{ PET scan } \\
\hline No & 1 (reference) & \multirow{2}{*}{0.25} \\
\hline Yes & 0.91 (0.77 to 1.07) & \\
\hline \multicolumn{3}{|c|}{$\begin{array}{l}\text { Analysis based on patients with complete set of information ( } \mathrm{n}=22770) \text {. } \\
\text { Adjusted hazard ratios and } 95 \% \text { confidence interval based on propensity } \\
\text { score analyses. Propensity scores obtained from first step model that } \\
\text { included age, sex, race, education, income, stage of disease, histology, } \\
\text { tumor size, surgery type, and lymph nodes resection as covariates. Cox } \\
\text { proportional hazards regression used as second step model. }\end{array}$} \\
\hline
\end{tabular}

regional (that is, appearing in metastases to cervical lymph nodes). ${ }^{1718}$ Neck ultrasound is the optimal imaging modality for detecting recurrence of regional thyroid cancer, and our previous work demonstrates a significant rise in the use of neck ultrasound in patients with thyroid cancer between 2001 to 2009. ${ }^{618}$ However, unlike distant metastases, the prognostic significance of regional recurrence for survival is debated. ${ }^{24}$ Therefore, it is plausible that greater use of ultrasound imaging is detecting more regional recurrence, but that this regional recurrence might not always indicate worse survival.

Use of radioiodine scans was associated with a significant increase in the use of surgery, radioactive iodine treatment, and radiation to treat recurrence. Radioiodine scans were associated with a significant improvement in disease specific survival. There are two plausible reasons for this finding. Radioactive iodine scans should only be used for patients with a history of iodine avid disease who have biochemical evidence of recurrence. These patients have a need to know whether structural iodine avid recurrence exists, and therefore warrant treatment with radioactive iodine. In patients with iodine avid disease, especially those with distant metastases that are not amendable to surgery, radioactive iodine is often effective treatment. In addition, iodine avidity generally suggests well differentiated disease, disease that historically is slower growing, and disease with a better prognosis. ${ }^{125}$ Even with propensity score analysis to reduce potential bias induced by confounding by indication, the relation between radioiodine scans and improved survival exists.

PET scans were also associated with a significant increase in the likelihood of being treated with surgery, radioactive iodine, and radiation after recurrence. However, PET scans were not associated with improved disease specific survival. There are two possible explanations for this finding. Firstly, disease detected by PET scans is rarely iodine avid and is typically less well differentiated, more aggressive, and less responsive to treatment than iodine avid disease. ${ }^{2627}$ Thus, PET positive disease correlates with worse prognosis, which could explain why use of PET imaging is not associated with improved disease specific survival. However, propensity score analysis, which introduces quasi-randomization, still showed no significant association between use of PET scans and outcome. Secondly, PET scanning is an expensive imaging modality, with a marked rise in use for patients with thyroid cancer since 2004. ${ }^{69}$ However, despite being used more frequently in these patients, our previous work has found that imaging with PET scans only changed management $30 \%$ of the time..$^{28}$ This suggests that although there is a role for PET scans in thyroid cancer surveillance, its use needs to be better defined.

\section{Strengths and limitations of study}

There is currently little data on effective long term surveillance in patients with thyroid cancer. A major strength of our study was that we evaluated current imaging practices after primary treatment of thyroid cancer and we investigated its relation to both recurrence and disease specific survival. A second strength is that we addressed potential confounding by indication through use of propensity score analysis.

This study had several limitations. Large patient databases, such as SEER-Medicare, lack patient specific data such as iodine avidity, patient preference, and indications for imaging or procedures. In addition, SEER-Medicare does not have adequate information on type of insurance and type of hospital. These could be relevant confounders that were unmeasured in our study. However, the large size of the SEER-Medicare database is also an asset because it allows us to identify associations that cannot be identified with smaller cohorts.

Another limitation was the use of billing data to identify imaging after primary treatment and treatment of recurrence. Although studies using billing data have limitations, previous studies focusing on other medical conditions have shown the feasibility and use of billing data to identify patients with specific diagnoses or procedures..$^{29-31}$ An additional limitation was that some physicians might use bedside ultrasound as an extension of the physical examination and a billing record might not be created. Therefore, the number of neck ultrasounds could have been underestimated. Physicians might also be more likely to get formal imaging if there is biochemical evidence of recurrence, such as rising thyroglobulin, or an abnormality on bedside ultrasound, thus resulting in a selection bias and inflating the likelihood that patients undergoing imaging will result in a diagnosis and treatment for recurrence.

Furthermore, this current study included patients primarily over the age of 65 years, which could have limited the applicability to other patient populations. However, the fastest increase in thyroid cancer has been seen in this age group. ${ }^{32}$ This cohort also has an increased risk of mortality from thyroid cancer due to their advanced age, which makes it the optimal cohort to identify any effect on survival. ${ }^{20}$ Finally, the retrospective nature of this study only allows us to show 
associations between imaging and treatment for recurrence or survival. Although causality could be complex, this study still serves as the necessary groundwork for future randomized controlled trials, cost effectiveness analysis, and studies assessing the role of patients in surveillance decision making.

Despite the study limitations, we believe that this study has powerful implications. As far as we know, it is the first population based study in differentiated thyroid cancer to assess the effect of imaging after primary treatment on treatment for recurrence and disease specific survival.

\section{Comparison with other studies}

Previous work has looked at the effect of imaging after primary treatment on survival in other cancers. ${ }^{33-35}$ In these other studies, more imaging was associated with a worse prognosis. However, in thyroid cancer, we found no strong relation between imaging and disease specific survival, except with radioiodine scans. Compared with other cancers, this finding might be due to the relatively indolent course of differentiated thyroid cancer versus other malignancies, or due to controlling for other patient characteristics (such as age and stage at diagnosis).

\section{Conclusions and policy implications}

This current study has implications for patients, physicians, and policy makers. It is the foundation for understanding when to image and when to treat recurrence, two key issues in the follow-up of patients with thyroid cancer. This controversy over appropriate imaging and subsequent management of recurrence arises from the overall excellent prognosis for patients with thyroid cancer and the fact that treatment of recurrence is not without risks and can result in patient harm. ${ }^{111-1620}$ In addition, although the incidence of thyroid cancer has been rising for the past several decades, the disease specific survival in differentiated thyroid cancer has remained relatively stable by comparison. ${ }^{1}$

Imaging after primary treatment could lead to more treatment for recurrence; however, without an improvement in survival, except for radioiodine scans in presumably iodine avid disease, it is not clear whether more imaging equals better care. It is also not clear whether the benefits of greater imaging outweigh the financial costs, heightened patient anxiety, and risk of patient harm from the treatment for recurrence. ${ }^{36-39}$ This current study is the foundation needed to define the appropriate long term follow-up of patients with thyroid cancer. This is the groundwork for future cost effectiveness studies, randomized controlled trials, and studies assessing the role of the patient and the physician in determining the optimal surveillance plan. The necessary next steps are to understand the role of patient preference and patient anxiety in surveillance trends, determine the role of physician incentives in defining imaging patterns, and determine patient outcomes when small recurrences are followed versus when small recurrences are intervened on.
David Hughes (Department of Surgery, University of Michigan), Barbra Miller, (Department of Surgery, University of Michigan), and Shruti Jolly (Department of Radiation Oncology, University of Michigan), assisted with identifying CPT codes for recurrences.

Contributors: MRH, JLW, and MB conceived and designed the study. CG carried out statistical analysis, with input from $M B$ and $M R H$. MRH, $M B$, and JLW wrote the manuscript, and all authors (MB, JLW, CG, BG, $\mathrm{MRH}$ ) contributed to manuscript critical appraisal and review. MB and $\mathrm{MRH}$ are the guarantors of this study. All authors had full access to all of the data, including statistical reports and tables, and take responsibility for the integrity of the data and the accuracy of the data analysis.

Funding: MRH is supported by National Institutes of Health grant 1 K07CA154595-02. MB is partly supported by grant 5 P30 CA 046592 from the National Cancer Institute. Support was provided by the Punya Foundation for Thyroid Cancer Research. The funder had no role in the design and conduct of the study; collection, management, analysis, and interpretation of the data; preparation, review, or approval of the manuscript; or the decision to submit the manuscript for publication.

Competing interests: All authors have completed the ICMJE uniform disclosure form at www.icmje.org/coi disclosure.pdf and declare: support from the Punya Foundation for Thyroid Cancer Research the submitted work; no financial relationships with any organizations that might have an interest in the submitted work in the previous three years; no other relationships or activities that could appear to have influenced the submitted work.

Ethical approval: Institutional review board approval was not required, because the study used publicly available data and did not contain personally identifiable information.

Data sharing: Statistical code is available from the first author at mousumib@umich.edu; dataset is available from SEER-Medicare at http://appliedresearch.cancer.gov/seermedicare/.

The lead author affirms that the manuscript is an honest, accurate, and transparent account of the study being reported; that no important aspects of the study have been omitted; and that any discrepancies from the study as planned (and, if relevant, registered) have been explained.

This is an Open Access article distributed in accordance with the Creative Commons Attribution Non Commercial (CC BY-NC 3.0) license, which permits others to distribute, remix, adapt, build upon this work non-commercially, and license their derivative works on different terms, provided the original work is properly cited and the use is non-commercial. See: http://creativecommons.org/licenses/ by-nc/3.0/.

1 Davies L, Welch HG. Current thyroid cancer trends in the United States. JAMA Otolaryngol Head Neck Surg 2014;140:317-22. doi:10.1001/jamaoto.2014.1.

2 Rahib L, Smith BD, Aizenberg R, Rosenzweig AB, Fleshman JM, Matrisian LM. Projecting cancer incidence and deaths to 2030: the unexpected burden of thyroid, liver, and pancreas cancers in the United States. Cancer Res 2014;74:2913-21. doi:10.1158/0008-5472. CAN-14-0155

3 Davies L, Welch HG. Increasing incidence of thyroid cancer in the United States, 1973-2002. JAMA 2006;295:2164-7. doi:10.1001/ jama.295.18.2164.

4 Sprague BL, Warren Andersen S, Trentham-Dietz A. Thyroid cancer incidence and socioeconomic indicators of health care access. Cancer Causes Control 2008;19:585-93. doi:10.1007/s10552-0089122-0.

5 Banerjee M, Muenz DG, Chang JT, Papaleontiou M, Haymart MR. Tree-based model for thyroid cancer prognostication. J Clin Endocrinol Metab 2014;99:3737-45. doi:10.1210/jc.2014-2197.

6 Wiebel JL, Banerjee M, Muenz DG, Worden FP, Haymart MR. Trends in imaging after diagnosis of thyroid cancer. Cancer 2015;121:1387-94. doi:10.1002/cncr.29210

7 Hollenbeak CS, Boltz MM, Schaefer EW, Saunders BD, Goldenberg D. Recurrence of differentiated thyroid cancer in the elderly. Eur 1 Endocrinol 2013;168:549-56. doi:10.1530/EJE-12-0848.

8 Tuttle RM, Tala H, Shah J, et al. Estimating risk of recurrence in differentiated thyroid cancer after total thyroidectomy and radioactive iodine remnant ablation: using response to therapy variables to modify the initial risk estimates predicted by the new American Thyroid Association staging system. Thyroid 2010;20:1341-9. doi:10.1089/thy.2010.0178.

9 Griffeth LK. Use of PET/CT scanning in cancer patients: technical and practical considerations. Proc (Bayl Univ Med Cent) 2005;18:321-30.

10 Huang B, Law MW, Khong PL. Whole-body PET/CT scanning: estimation of radiation dose and cancer risk. Radiology 2009;251:166-74. doi:10.1148/radiol.2511081300. 
11 Sawka AM, Thabane L, Parlea L, et al. Second primary malignancy risk after radioactive iodine treatment for thyroid cancer: a systematic review and meta-analysis. Thyroid 2009;19:451-7. doi:10.1089/ thy.2008.0392

12 Lang BH, Lee GC, Ng CP, Wong KP, Wan KY, Lo CY. Evaluating the morbidity and efficacy of reoperative surgery in the central compartment for persistent/recurrent papillary thyroid carcinoma. World J Surg 2013;37:2853-9. doi:10.1007/s00268-013-2202-7.

13 Terezakis SA, Lee KS, Ghossein RA, et al. Role of external beam radiotherapy in patients with advanced or recurrent nonanaplastic thyroid cancer: Memorial Sloan-kettering Cancer Center experience. Int J Radiat Oncol Biol Phys 2009;73:795-801. doi:10.1016/j. ijrobp.2008.05.012.

14 Tufano RP, Clayman G, Heller KS, et al. American Thyroid Association Surgical Affairs Committee Writing Task Force. Management of recurrent/persistent nodal disease in patients with differentiated thyroid cancer: a critical review of the risks and benefits of surgical intervention versus active surveillance. Thyroid 2015;25:15-27. doi:10.1089/thy.2014.0098.

15 Roh JL, Yoon $\mathrm{YH}$, Park Cl. Recurrent laryngeal nerve paralysis in patients with papillary thyroid carcinomas: evaluation and management of resulting vocal dysfunction. Am / Surg 2009;197:45965. doi:10.1016/j.amjsurg.2008.04.017.

16 Van Nostrand D. Sialoadenitis secondary to ${ }^{131}$ therapy for well-differentiated thyroid cancer. Oral Dis 2011;17:154-61. doi:10.1111/j.1601-0825.2010.01726.x.

17 Cooper DS, Doherty GM, Haugen BR, et al. American Thyroid Association (ATA) Guidelines Taskforce on Thyroid Nodules and Differentiated Thyroid Cancer. Revised American Thyroid Association management guidelines for patients with thyroid nodules and differentiated thyroid cancer [correction in: Thyroid 2010;20:942] Thyroid 2009;19:1167-214. doi:10.1089/thy.2009.0110.

18 Haugen BR, Alexander EK, Bible KC, et al. 2015 American Thyroid Association management guidelines for adult patients with thyroid nodules and differentiated thyroid cancer: the American Thyroid Association Guidelines task force on thyroid nodules and differentiated thyroid cancer. Thyroid 2016;26:1-133. doi:10.1089/ thy. 2015.0020 .

19 Smallridge RC, Diehl N, Bernet V. Practice trends in patients with persistent detectable thyroglobulin and negative diagnostic radioiodine whole body scans: a survey of American Thyroid Association members. Thyroid 2014;24:1501-7. doi:10.1089/ thy.2014.0043.

20 National Cancer Institute. Surveillance, epidemiology, and end results program. http://seer.cancer.gov/.

21 Fritz A, Percy C, Jack A, et al; eds. International classification of diseases for oncology. 3rd ed. World Health Organization, 2000.

22 Center for Disease Control and Prevention. National Center for Health Statistics. 2016 www.cdc.gov/nchs/.

23 D’Agostino RB Jr. Propensity score methods for bias reduction in the comparison of a treatment to a non-randomized control group. Stat Med 1998;17:2265-81. doi:10.1002/ (SICI)1097-0258(19981015)17:19.

24 Nixon IJ, Wang LY, Palmer FL, et al. The impact of nodal status on outcome in older patients with papillary thyroid cancer. Surgery 2014;156:137-46. doi:10.1016/j.surg.2014.03.027.
25 Song HJ, Qiu ZL, Shen CT, Wei WJ, Luo QY. Pulmonary metastases in differentiated thyroid cancer: efficacy of radioiodine therapy and prognostic factors. Eur J Endocrinol 2015;173:399-408. doi:10.1530/ EJE-15-0296.

26 Wang W, Larson SM, Fazzari M, et al. Prognostic value of [18F] fluorodeoxyglucose positron emission tomographic scanning in patients with thyroid cancer. J Clin Endocrinol Metab 2000;85:1107-13.

27 Schreinemakers JM, Vriens MR, Munoz-Perez N, et al. Fluorodeoxyglucose-positron emission tomography scan-positive recurrent papillary thyroid cancer and the prognosis and implications for surgical management. World J Surg Oncol 2012;10:192. doi:10.1186/1477-7819-10-192.

28 Wiebel JL, Esfandiari NH, Papaleontiou M, Worden FP, Haymart MR. Evaluating positron emission tomography use in differentiated thyroid cancer. Thyroid 2015;25:1026-32. doi:10.1089/thy.2015.0062.

29 Singh SM, Webster L, Calzavara A, Wijeysundera HC. Validation of algorithms to identify invasive electrophysiology procedures using administrative data in Ontario, Canada. Med Care 2014. doi:10.1097/ MLR.0000000000000274.

30 Butt DA, Tu K, Young J, et al. A validation study of administrative data algorithms to identify patients with Parkinsonism with prevalence and incidence trends. Neuroepidemiology 2014;43:28-37. doi:10.1159/000365590

31 Warren JL, Harlan LC, Fahey A, et al. Utility of the SEER-Medicare data to identify chemotherapy use. Med Care 2002;40(suppl):IV-55-61. doi:10.1097/00005650-200208001-00008.

32 Morris LG, Sikora AG, Tosteson TD, Davies L. The increasing incidence of thyroid cancer: the influence of access to care. Thyroid 2013;23:885-91. doi:10.1089/thy.2013.0045.

33 Hyder O, Dodson RM, Mayo SC, et al. Post-treatment surveillance of patients with colorectal cancer with surgically treated liver metastases. Surgery 2013;154:256-65. doi:10.1016/j.surg.2013.04.021.

34 Hyder O, Dodson RM, Weiss M, et al. Trends and patterns of utilization in post-treatment surveillance imaging among patients treated for hepatocellular carcinoma. J Gastrointest Surg 2013;17:1774-83. doi:10.1007/s11605-013-2302-6.

35 Witkowski ER, Smith JK, Ragulin-Coyne E, Ng SC, Shah SA, Tseng JF. Is it worth looking? Abdominal imaging after pancreatic cancer resection: a national study. J Gastrointest Surg 2012;16:121-8. doi:10.1007/ s11605-011-1699-z.

36 Boltz MM, Hollenbeak CS, Schaefer E, Goldenberg D, Saunders BD. Attributable costs of differentiated thyroid cancer in the elderly Medicare population. Surgery 2013;154:1363-9, discussion 1369-70. doi:10.1016/j.surg.2013.06.042.

37 Aschebrook-Kilfoy B, Schechter RB, Shih YC, et al. The clinical and economic burden of a sustained increase in thyroid cancer incidence. Cancer Epidemiol Biomarkers Prev 2013;22:1252-9. doi:10.1158/1055-9965.EPI-13-0242.

38 Misra S, Meiyappan S, Heus L, et al. Patients' experiences following local-regional recurrence of thyroid cancer: a qualitative study. J Surg Oncol 2013;108:47-51. doi:10.1002/jso.23345.

39 Bresner L, Banach R, Rodin G, Thabane L, Ezzat S, Sawka AM. Cancer-related worry in Canadian thyroid cancer survivors. J Clin Endocrinol Metab 2015;100:977-85. doi:10.1210/jc.2014-3169.

(c) BMJ Publishing Group Ltd 2016 